\title{
La andragogía y la formación de emprendedores en la UNESR Bolívar
}

Recibido: 7 de abril de 2019

Aceptado: 20 de mayo de 2019

Publicado: 24 de junio de 2019

\author{
Marcel Doubront \\ marceldoubront@gmail.com \\ Universidad Nacional Experimental \\ Simón Rodríguez (Venezuela)
}

Resumen: Ante la compleja crisis que experimenta Venezuela, que ha provocado una sistemática deserción de los estudiantes universitarios, surge la necesidad de estructurar un plan de formación para emprendedores, que permita crear nuevas empresas, donde la juventud tenga un papel protagónico, fortaleciéndose una cultura de emprendimiento compatible con la filosofía andragógica, participativa y flexible de la Universidad Nacional Experimental Simón Rodríguez (UNESR).

Palabras clave: Educación, universidad, sociedad, crisis venezolana, emprendimiento.

Abstract: Given the complex crisis experienced by Venezuela, which has provoked a systematic defection of university students, there is a need to structure a training plan for entrepreneurs, to create new businesses, where youth have a leading role, strengthening a culture of entrepreneurship compatible with Andragogical, participatory and flexible philosophy of Universidad Nacional Experimental Simón Rodríguez (UNESR).

Key words: Education, University, Society, Venezuelan Crisis, Entrepreneurship.

\section{Introducción}

En la actualidad, ante la configuración de un mundo globalizado, se hace necesaria la búsqueda de alternativas ante los desafíos económicos y sociales. A través del tiempo y la historia, tanto los medios como las relaciones de producción han ido variando, producto de los contextos en donde se vive. Como ejemplo se puede tomar la sociedad primitiva, la cual vivía de la recolección, la caza y el intercambio. Tiempo después, en la época colonial, las relaciones de intercambios fueron variando a través de la aparición del valor monetario lo que sin duda hizo que se desvirtuara el antiguo concepto de comunidad.

En el siglo XVIII se produce la revolución industrial que sustituyó no solo el trabajo manual o artesanal por la industrialización (de distintos rubros productivos bien sea textiles, alimenticios, entre otros), sino que estableció transformaciones en el contexto mundial, sea en lo económico, político, social y cultural. Entre dichas transformaciones se hace referencia al capitalismo como ideología dominante, donde se pudieron observar cambios no solo tecnológicos, sino también otros factores que perviven hasta nuestros días, tales como el mal uso de los recursos naturales, la explotación y la reducción de mano de 
obra; competencias desleales y desiguales entre medios de producción y comercialización. En ese contexto, surge lo que se conoce como "emprendimiento", el cual es

un mecanismo clave para el crecimiento económico y el desarrollo de la innovación de una región. La fundación de nuevas empresas va asociada a la generación de empleo, la creación de riqueza, el incremento de la competitividad y el desarrollo tecnológico (Vallmitjana, 2014, p. 17).

Ante la dinámica económica mundial (el cual no solo influye en lo económico, sino en lo político y social), el emprendimiento representa una alternativa ante los grandes desafíos globales, abarcando la creación de nuevos medios de producción, distribución y comercialización, hasta la de nuevos productores, empleados y productos, con una nueva geometría económica más humanizada.

\begin{abstract}
El emprendimiento es una disciplina científica relativamente joven que ha experimentado un crecimiento substancial en los últimos años, tanto en EUA como en países europeos. Este aumento de los estudios sobre la actividad emprendedora va asociado a la progresiva relevancia de la contribución de las nuevas empresas en las economías de los territorios (Vallmitjana, 2014, p. 17).
\end{abstract}

\title{
2. Sobre el emprendimiento
}

El emprendimiento aparece como una alternativa ante el alto índice de desocupación y desempleo, sobre todo en los segmentos más jóvenes de la población.

Los jóvenes y las mujeres son los más perjudicados en el ámbito laboral latinoamericano, según el estudio Panorama Laboral 2018 publicado por la Organización Internacional del Trabajo (OIT). Uno de cada cinco jóvenes de 15 a 24 años de la región el 19.6 por ciento busca trabajo y no encuentra, una cifra que casi triplica la tasa de desempleo entre los mayores de 25 años 6.3 por ciento (Fowks, 2018).

Los problemas relacionados con el desempleo juvenil exigen la implementación de políticas destinadas a mejorar las oportunidades de trabajo dentro del mercado laboral. Entre las iniciativas que destacan, aparecen las siguientes:

- Hacer de la generación de empleo juvenil una prioridad en la agenda del diálogo social entre los actores fundamentales de la economía.

- Apoyar el espíritu emprendedor de los jóvenes para que pongan en práctica sus propias iniciativas a través de sistemas de micro crédito como "incubadoras de empresas".

- Dar eficiencia y cobertura a los servicios de empleo, sitios digitalizados, oficinas donde se dé a los jóvenes información en tiempo real sobre posibilidades inmediatas de enganche.

- Debatir sobre la educación necesaria para que se articule mejor con el mercado laboral, estimule la innovación, recalifique la mano de obra y facilite la certificación de competencias.

- Incrementar los sistemas de pasantías para consolidar la formación profesional de los jóvenes en las empresas y el sector público y facilitar la transición educación-trabajo. 
- Dar acceso a los jóvenes a un sistema de créditos educativos, transferencias monetarias condicionadas y becas sueldo para que puedan continuar su formación y recalificación laboral.

- Facilitar que las mujeres jóvenes se mantengan en el mercado laboral, a través de guarderías para sus hijos y turnos de todo el día en las escuelas (OIT, 2017).

\section{Papel de la universidad: educación de pertinencia}

Dichas recomendaciones guardan estrecha consonancia con el objeto de estudio, ya que estas referencias coinciden en la necesidad de buscar un plan de formación para emprendedores que permita crear nuevas empresas (donde la juventud tenga un papel protagónico) y que esta cultura de emprendimiento incida en los factores económicos, políticos, sociales y culturales, los cuales afectan significativamente a la juventud venezolana, haciendo que estos deserten de sus estudios universitarios.

La crisis económica que atraviesa el país está afectando abundantemente a la Universidad del Zulia, al igual que a muchas otras de las universidades, tanto públicas como privadas de Venezuela. Esta actual situación del país ha llevado a que se produzca una numerosa deserción de estudiantes. Muchos argumentos se han dado para explicarla: la crisis económica, en donde los costos de vida son insostenibles, les imposibilita poder responder a los gastos necesarios para su alimentación, transporte, residencia, en especial para estudiantes foráneos, quienes componen gran parte de la población estudiantil, además, afecta el rendimiento académico de estos estudiantes y hace que abandonen la carrera o bajen de manera demostrativa su rendimiento académico (Ferrer, Urribarrí \& Vera, 2017, p. 15)

Los impactos de esta crisis inciden en el bajo rendimiento académico, en la deserción estudiantil de las universidades bien (temporales o permanentes), así como el preocupante índice migratorio de venezolanos a países vecinos.

\footnotetext{
Venezuela, actualmente, se encuentra sumida en una situación política y económica difícil, debido a la concurrencia de determinados problemas sociales que inciden en el bienestar individual y colectivo de la población. El aumento de los niveles de pobreza, como consecuencia del incremento de la tasa de inflación y la falta de divisas suficientes por la reducción de los precios del petróleo, ha ocasionado un proceso de migración atípico en la población venezolana, especialmente, en los individuos más jóvenes, quienes agobiados por la falta de oportunidades laborales y la insatisfacción de sus necesidades básicas, deciden salir del país (Albarrán, 2019, p. 60).
}

Tal situación no puede ni debe ser indiferente para las universidades, ya que las casas de estudios están al servicio de la nación y deben colaborar en la orientación de la vida del país mediante su acción formativa.

Las transformaciones políticas que está viviendo el país han puesto a la universidad pública ante la necesidad de reflexionar sobre sí misma, sobre su lugar en la producción de interpretaciones culturales de la realidad, sobre su papel de mediadora cultural en las relaciones entre Estado y sociedad y sobre la naturaleza de su carácter público (De Sousa, 2007, p. 7). 
En esta situación crítica del país, se requiere de una "educación de pertinencia", que pueda hacer frente a los grandes desafíos: que motive e involucre a los diferentes actores de la sociedad (la juventud profesional o en proceso de formación) a cumplir el papel que les corresponde. Sin embargo, dicha aspiración sería totalmente imposible si la educación sigue manteniendo un lenguaje antagónico a las expectativas del educando. Con relación a lo planteado, Gutiérrez (1974) hacía referencia a

una educación cuyo objetivo sea el desarrollo de la vida cultural tendrá objetivos y metodologías muy diferentes, y hasta opuestos, a los de la educación que tienda hacia una civilización tecnológica. Basta comprobar que en la actual pedagogía los educandos dentro del aula de clases, viven divorciados de la propia cultura (p. 14).

En analogía con lo que se plantea en el párrafo anterior, Maturana (1992) sostiene que "el mundo en que vivimos lo configuramos en la convivencia, incluso cuando hablamos de lo interno y lo externo [...] el mundo en que vivimos es el mundo que nosotros configuramos y no un mundo que encontramos" (p. 30), lo que sin duda alguna representa una gran oportunidad (desde el contexto universitario) establecer un cimiento de desarrollo humano, económico, cultural y ciudadano desde la formación de nuevos y pertinentes profesionales.

La formación para un profesional de pertinencia debe estar orientada a la visión y análisis de los diversos aspectos y acontecimientos que rigen a la actual sociedad, ya que de lo contrario se seguirá conservando un divorcio entre el mundo académico y la realidad social, en sintonía con este planteamiento, Herrera (2019) afirma que "no puede existir una sociedad del conocimiento en la que haya un divorcio entre la universidad, la sociedad, la empresa privada y el sector público" (p. 392). Por ello, este tipo de reflexiones deben llevar al entendimiento de la necesidad de una universidad de contenido multidisciplinario, a fin de que su visión prospectiva conlleve al esclarecimiento de los problemas sociales que afronta el país.

\footnotetext{
Los abundantes diagnósticos de la crisis universitaria, en Venezuela y el mundo, dan cuenta de múltiples dimensiones en donde se constata la inviabilidad de un modelo epistemológico, pedagógico y organizacional que ya no se corresponde más con las expectativas de los nuevos actores que emergen en la escena, con las exigencias de pertinencia social y participación popular (Fergusson \& Lanz, p. 2).
}

Al hacer referencia a los procesos de transformación o vencimiento de los tradicionales paradigmas, es necesario reflexionar sobre la misión de las casas de estudios en aras de entender los aciertos y desaciertos de la gestión formativa que se emprende. Ortega y Gasset (2001) hace mención que "una institución no puede constituirse en buenos usos si no se ha acertado con todo rigor al determinar su misión".

El presente estudio toma como delimitación institucional a la Universidad Nacional Experimental Simón Rodríguez, la cual es definida por Caraballo (2012) como "una institución de Educación Superior venezolana pública, pionera en la aplicación de la andragogía como modelo educativo en la formación y capacitación de profesionales en las carreras de educación, administración e ingeniería de alimentos". 


\title{
4. La andragogía y la problemática educativa
}

La andragogía hace referencia a una educación orientada al adulto, en contraposición de la pedagogía, entendida como una educación orientada a los niños (paidós). Alcalá (2012) sugiere que la andragogía es una "praxis fundamentada en los principios de participación y horizontalidad” (p. 5), lo cual deja ver claramente que la andragogía es una educación al adulto, con adultos y entre adultos, por lo que la acción educativa no se traduce en una transmisión de contenidos, sino en la reflexión y transformación de los mismos.

\begin{abstract}
La andragogía se centra en el aprendizaje, no en la enseñanza. Más que un proceso de "recorrer" una serie de pautas y unidades curriculares, la andragogía tiene que flexibilizar los modos de llevar a cabo un proceso de formación, de generar situaciones que favorezcan al máximo el aprendizaje entre las personas. El sentido que tenga el aprendizaje para las personas es más importante que la acumulación de unidades crédito. Ello implica que la formación ha de ser un proceso permanentemente creativo, significativo y vital, y las instituciones educativas tienen que transformarse para que eso sea posible (Valdez, 2010, p. 61).
\end{abstract}

Al consultar a la comunidad universitaria de la UNESR, el estudio realizado por Caraballo (2012), recogió una "multiplicidad de acepciones" de la andragogía, las cuales

\begin{abstract}
repercuten considerablemente en la práctica educativa del facilitador de la Institución, debido a que no existe una postura clara que guíe y oriente cómo deben ser los procesos de enseñanza y aprendizaje en la práctica, sobre todo si se consideran los cambios estructurales que han surgido en el contexto educativo de la UNESR a raíz de la masificación de la enseñanza, la aparición y desarrollo progresivo de las TIC, y la educación permanente y a lo largo de toda la vida. Se hace necesario pues, dotar a la andragogía de una dimensión clara y coherente que permita vincularla con la realidad en el contexto actual de la UNESR.
\end{abstract}

¿Cómo puede existir una correspondencia de las universidades con la sociedad, si esta no está en sintonía con su filosofía? En virtud de lo expuesto, Gutiérrez (1974) afirma que "la andragogía implica, por lo tanto, una nueva actitud del hombre frente al problema educativo. La educación de adultos no puede quedar reducida a la escolarización de la sociedad" (p. 80). Si la educación quiere transcender a los problemas sociales, esta no puede seguir descontextualizada de la realidad social.

el problema de la educación no puede ser considerado aisladamente del contexto político, social, económico y cultural en que se da. Hoy es un lugar común de la sociología de la educación con el mismo sistema social vigente en todo país la educación responde o es reflejo del tipo de sociedad existente (Ander Egg, 1977, p. 13).

Al querer buscar una alternativa educativa a los complejos desafíos que hoy se presentan en la sociedad, es necesario que la universidad (entre ellas la UNESR) tenga claro sus preceptos filosóficos, a fin que su estrategia no solo sea compatible con las demandas de la sociedad, sino con ella misma. En ese sentido, la UNESR (2009) planteó que "el proceso de transformación universitaria está condicionado a introducir transformaciones profundas en la dimensión curricular, entendida, como la integración de los procesos formativos de 
extensión, investigación y producción". Empero, hasta la fecha, dicho planteamiento no ha sido posible, puesto que no se puede formar en la llamada "sociedad del conocimiento" estudiantes del siglo XXI con pensamiento y estrategias del siglo pasado

La vieja universidad no es adaptable a la nueva época en cuyo tránsito se han roto todas las centralidades, se desvanecen los viejos mitos, se relativizan las antiguas hegemonías" (Fergusson \& Lanz, 2011, p. 3).

Sería muy fácil proponer un diplomado de emprendimiento (con un conjunto de módulos, contenidos y letrados que atiendan el desafío del emprendimiento) como una respuesta coyuntural ante la situación económica que vive el país. Sin embargo, el problema es más complejo y no puede abordarse como una situación de forma, sino como una oportunidad para construir una nueva geometría social, económica, política, cultural y ciudadana, asegurándose un desarrollo sostenible.

\section{Formación del emprendimiento}

En términos concretos, el emprendimiento puede considerarse como una visión motivada por diversas circunstancias que determinan el cumplimiento de una meta u objetivo. Desde el punto de vista económico y empresarial, el emprendimiento

es un estímulo descentralizado que implica facilitar el desarrollo de iniciativas económicas y sociales. Asimismo, está esencialmente vinculado con la concepción de desarrollo endógeno, el uso de redes como organización para aprovechar el conocimiento y la práctica innovadora colectiva de una sociedad que procura configurar nuevos espacios potencialmente innovadores (Petit, 2007).

El emprendimiento nace producto de una necesidad, en la cual el sujeto se motiva y participa en los procesos de cambios sociales y educativo-culturales, persiguiendo la adquisición y transformación del conocimiento, a fin de crear su propia empresa. Abad (2014) explica que el emprendimiento "es una actividad que aporta a superar las crisis económicas, dado que potencia la actividad económica, promueve el intercambio y requiere de mano de obra”. Como una alternativa para la superación de las crisis económicas,

el emprendimiento implica un autoempleo, el emprendedor genera una actividad económica que lo extrae del desempleo, incorporando en la actividad económica a otros agentes demandantes de mano de obra y recursos que potencien el auge económico (Abad, 2014).

En el contexto venezolano, el emprendimiento surge de manera significativa por la carencia de empleo, la insuficiencia de los sueldos y salarios. Raffino (2019) evalúa las características que debe tener una persona emprendedora.

- Innovadora: debe crear un proyecto a partir de ideas nuevas y originales.

- Creativa: una idea poco interesante puede no llegar a generar resultados óptimos.

- Capacidad de asumir riesgos: debe superar los retos que el desarrollo de un proyecto pueda generar. 
- Perseverante: en muchos casos (quizá en la mayoría), las ganancias no llegan en los primeros meses, sino tiempo después de comenzado el emprendimiento.

- Flexibilidad y adaptabilidad: siempre focalizado en crecer.

Ahora bien, ¿Cómo convencer tanto a la academia como a la institución de una formación para el emprendimiento? En la mayoría de los casos, el emprendimiento surgió de la búsqueda de una alternativa a la situación económica (que conlleve el crecimiento personal del emprendedor), a través de la conformación de empresas con fines lucrativos o no. Sin embargo, las acciones ejercidas de estos innovadores no suelen nutrirse de un conocimiento técnico basado en la planificación, organización, dirección y control que permita darle sostenibilidad. Si bien la iniciativa fue forjada como vía de solución o respuesta al desafío presentado, es insuficiente la buena intención cuando se carece de solidez formativa.

\section{Contexto venezolano}

Paralelo a la migración de profesionales y estudiantes al extranjero, la crisis económica ha producido un aumento progresivo de pequeños productores y comerciantes, quienes recurren a la fabricación de productos artesanales (jabones, harina de maíz, margaría, entre otros), los cuales en muchos casos suplen los artículos de primera necesidad producidos por los grandes medios de producción.

\footnotetext{
Situación similar ocurre en Venezuela, donde el aumento del desempleo, así como la depreciación del aparato productivo, indica que las pequeñas y medianas empresas son una buena alternativa para todas aquellas personas que entran al mercado laboral. Sin embargo, por el camino que deben recorrer se puede pronosticar poco éxito debido al exceso de trámites administrativos, así como también complicaciones burocráticas, la disminución en el poder adquisitivo, la vulnerabilidad de la propiedad privada, la falta de apoyo a la inversión, la inseguridad económica, los altos niveles de inflación y la devaluación de la moneda, son elementos influyentes en dicha práctica (Del Valle, Meriño \& Martinez, 2018, p. 117).
}

De acuerdo con Felix Ríos, en Venezuela "existe una alta tasa de actividad emprendedora temprana. Más de dos millones y medio de personas inician un emprendimiento en un lapso de 0 a 3 años, pese a la situación país" (Universidad Arturo Michelena, 2019). Sin embargo, Rodríguez (2014) precisa que

entre el 18\% y el 20\% de los venezolanos mayores de edad están iniciando un nuevo negocio. Todos los años se ve eso... y cuando vemos el porcentaje de emprendimientos que pasan de los tres años y medio es alrededor del $1 \%$ o del $1.5 \%$. Es decir, tienes $20 \%$ de personas emprendiendo y a su vez tienes $1.5 \%$ de personas con negocios con más de tres años y medio establecidos... Hay una brecha súper fuerte allí entre los que nacen y los que se mantienen después de los 3.5 años.

Aunque es conocido el poco nivel de prosecución de estos nacientes empresarios (producto de las condiciones adversas como limitantes para adquirir divisas, materias primas, malos servicios públicos, alto nivel inflacionario, carencia de seguridad jurídica, física y emocional) continúa incrementándose la cantidad de emprendedores. 
Llama poderosamente la atención que aun con todos estos factores en contra del emprendimiento, los venezolanos toman el riesgo de realizar iniciativas de negocios, esto se debe a la necesidad económica que poseen, impulsados por las mismas condiciones del entorno, impulsados por la ausencia de empleo, la necesidad de crear ingresos para su sostenimiento, lo que motiva a aprovechar oportunidades en el momento preciso, no garantizándoles el éxito, todo esto movido por mantener una estabilidad económica (Del Valle, Meriño \& Martinez, 2018, p. 118).

Ahora bien, emprender no es simplemente montar un negocio, crear y vender un producto. Todo este proceso requiere un entendimiento y una visión prospectiva de lo que se quiere lograr. Rodríguez (2014) refiere que "muy pocos emprendimientos en Venezuela son dados a la innovación, ya que la mayoría de los emprendimientos te dicen que nacen empresas o desarrollan empresas que prácticamente hacen lo mismo". No necesariamente el emprendedor es quien posee un medio de producción: también es el que puede establecer óptimas relaciones de producción para la distribución y colocación estratégica de los productos. Así, un emprendedor

debe buscar un modelo de negocio escalable, con potencial de crecimiento de miles por ciento, algo que cubra grandes necesidades, que resuelva problemas. Las ideas que logran ser disruptivas son aquellas que ven el futuro, no solo fijarse como se ha hecho hasta ahora sino que puede cambiar esa dinámica de mercado. No es buscar hacer dinero y ya, es cambiar mi sector para que el dinero venga (Benavente, 2018).

Esta visión es necesaria para brindar sostenibilidad a las empresas formadas por los emprendedores, apostándose por su crecimiento y desarrollo. Si un emprendedor no cuenta con visión ni conocimiento sobre lo que promueve su actividad, no obtendrá los resultados esperados. Rodríguez (2014) advierte del "corto tiempo que suelen mantenerse la mayoría de los emprendimientos en Venezuela" por la ineficiente estructura macroeconómica y la falta de formación adecuada para emprender: "tener un título universitario no implica que la persona está preparada para iniciar su propio negocio”. Sin embargo, Rodríguez (2014) destaca que cada vez hay mayor preocupación en las universidades por adelantar programas de apoyo al emprendimiento".

Si la educación quiere repercutir en la transformación de los desafíos que hoy adversamente afronta la sociedad venezolana, la universidad debe romper con su tradicional burocracia conservadora y convertirse en un aliado o compañero de una lucha común para el logro del desarrollo y progreso del país.

\footnotetext{
"Educar para el emprendimiento" surge como una necesidad ante los tiempos que vive el país, en primer lugar en los extractos sociales más bajos como en tiempos de la IV República se evidencia un alto nivel de deserción escolar tanto en la educación media y universitaria, como de igual manera preocupantes indicadores como el de embarazo no planificado en adolescentes y delincuencia juvenil, todo esto no solo por los niveles socio económicos ya conocidos, sino porque la educación no genera ninguna atracción para la juventud ya que no da respuesta a sus expectativas (Doubront, 2017).
} 
Lo planteado permitirá superar los alarmantes índices de pobreza y miseria (generado por el alto índice inflacionario), así como el vencimiento del asistencialismo y la cultura rentista que por décadas se convirtió en un factor contaminante para la sociedad venezolana.

Es imperante que la estructura magistral internalice los frutos del emprendimiento o que por lo menos los visibilice, a la par que la innovación sea el garante para que las iniciativas diversas tengan asiento desde la fundamentación de la investigación como componente transversal de quien se forma y termina egresando de las instituciones. Es de apreciar, que dicho proceso ha sido estimado por fuera del alcance de la ruta académica magistral (Correa, Delgado \& Conde, 2011, p. 146).

Lo expuesto destaca la importancia de una educación para el emprendimiento y la pertinencia de una cultura de la innovación y la empresarialidad, evitándose que de las casas de estudios no egresen simples letrados con licencias para un determinado oficio o empleados con visión reduccionista, sino graduar productores, empleadores, lideres, coadyuvantes de la construcción de una nueva y mejor sociedad.

Es necesario establecer la importancia de la cultura de innovación para impulsar el desarrollo desde adentro. Uno de los factores propios de la sociedad del conocimiento es la omnipresencia de la cultura de la innovación como forma de establecer el crecimiento sostenido a largo plazo (Petit, 2007).

Lo referido no representa una utopía. Por el contrario, según la ley de universidades venezolana, en su Artículo 2, se indica que "las Universidades son instituciones al servicio de la nación y a ellas corresponde colaborar en la orientación de la vida del país mediante su contribución doctrinaria en el esclarecimiento de los problemas nacionales", por lo que este planteamiento es una responsabilidad a cumplir.

\section{A modo de conclusión: estructurar una alternativa educativa en emprendimiento}

Lo planteado deja otra interrogante: ¿cómo establecer una alternativa formativa para la creación de profesionales con una visión en emprendimiento?

En primer lugar, considerar que la "educación para la vida" contempla el desarrollo humano del educando desde el plano individual y también como actor o coadyuvante de los procesos de desarrollo en la sociedad. La formación, como hecho colectivo, permite adquirir y construir los saberes para transformarlos: "frente a los numerosos desafíos del porvenir, la educación constituye un instrumento indispensable para que la humanidad pueda progresar hacia los ideales de paz, libertad y justicia social" (Delors, 1996).

Al abordar los complejos desafíos que afronta la realidad venezolana, es pertinente brindar desde las universidades (sobre todo las que forman educadores, administradores e ingenieros), herramientas para contribuir con los cimientos de desarrollo local, regional y nacional, en aras de transformar la delicada realidad económica y social que hoy abruma al país. 
La formación de emprendedores y nuevas empresas es una prioridad cuando se trata de promover el desarrollo económico y social de los países, por ello es necesario determinar cuál es su contribución a estos objetivos. El emprendimiento resulta importante por sus efectos positivos en la generación de empleos y en el crecimiento económico (Zamora, 2017).

No obstante, las reflexiones que se plantean no están enfocadas únicamente hacia el desarrollo económico, sino a la búsqueda de una transformación política, cultural y ciudadana de la República Bolivariana de Venezuela. Según Cruz Diez, "hay que inventar un nuevo lenguaje político que hable de democracia, de valores éticos, de libertad, progreso y justicia social, hay que inventar la educación y crear un país de emprendedores, artistas e inventores, un país digno y soberano" (Panorama, 2017). Tal aspiración no llegará desde la casualidad o desde la ilusión, sino a través del conocimiento técnico promovido por la universidad en sus planes de estudios, una andragogía de compromiso multidisciplinario con planes de formación para el emprendimiento productivo.

Es de vital importancia promover desde las instituciones de educación universitaria (antes de la aplicación de cualquier programa formativo) la cultura de emprendimiento desde una perspectiva multi y transdisciplinaria, lo cual está en consonancia ante la sobreoferta de licenciados (que supera la demanda de personal), el clima de incertidumbre del país y los bajos sueldos. Una nueva visión (basada en la innovación) permitirá la creación de un nuevo perfil del egresado universitario. Las casas de estudios deben articular su proceso de formación con los medios de producción y las necesidades de la comunidad, con la finalidad de que el educando tenga una visión más consonante con la realidad.

Si pudiéramos entender la importancia de la universidad como centro del saber, como el lugar donde se genera el conocimiento de la humanidad, dejaríamos de pensar que la culminación de una carrera es simplemente el cumplimiento de los contenidos analíticos de una malla curricular, o que un docente es experto en su materia porque simplemente sabe "lo de la materia" (González Velasco, 2012, p. 11).

La visión de este nuevo profesional emprendedor debe tener sustento ético, a fin de no recaer en la explotación, la especulación, el acaparamiento y la inflación de los precios de los productos por la poca oferta de los artículos de consumo.

El emprendimiento ha tenido un innegable reconocimiento y trascendencia en las sociedades contemporáneas, dada su importancia en el desarrollo económico y social de las regiones, sin embargo, en los procesos de enseñanza a nivel mundial se han orientado de manera privilegiada a la creación de riqueza a partir de la generación de empresas e ideas de negocio, al margen de un proceso de formación donde se desarrolle mediante la creatividad, el trabajo en equipo, el planteamiento o la solución de problemas por parte de las personas (Del Valle, Meriño \& Martinez, 2018, p. 117).

Una educación de emprendimiento productivo, orientado desde la andragogía, representa una alternativa al tradicional modelo formativo y la innovación de una construcción del saber colectivo de visión compleja y transcompleja. Gibb afirma que "la educación en emprendimiento demanda de la integración del conocimiento de varias disciplinas y 
mayores oportunidades de desarrollar espacios de aprendizaje experiencial y la necesidad de desarrollar de manera apropiada comportamientos, actitudes y habilidades emprendedoras" (Correa, Delgado \& Conde, 2011, p. 42).

Con un nuevo modelo didáctico y visión prospectiva, la innovación permitirá ser la contraparte al pensamiento promovido por la instrucción tradicional. Una educación para el emprendimiento, desde la andragogía, auspicia una visión holística de la sociedad.

Yo creo profundamente que cuando menos mutilante sea un pensamiento, menos mutilará a los humanos. Hay que recordar las ruinas que las visiones simplificantes han producido, no solamente en el mundo intelectual, sino también en la vida. Suficientes sufrimientos aquejaron a millones de seres humanos como resultado de los efectos del pensamiento parcial y unidimensional (Morín, 1998, p. 118).

Raffino (2019) expone que "un emprendimiento da independencia. Una persona que posee un emprendimiento propio no depende de jefes ni horarios ya que trabaja de manera particular". El emprendimiento ofrece libertad, autonomía y oportunidad de crecimiento. Empero, el poco conocimiento para formar y desarrollar una empresa puede incrementar las dificultades de estas iniciativas.

la creación de nuevas empresas es considerada como uno de los factores determinantes para el crecimiento económico y la competitividad de los países, mejorando el nivel de vida de los de hecho, su relación con la educación ha sido ampliamente estudiada por investigaciones internacionales, desde distintas perspectivas y enfoques, apareciendo estudios que encuentran una relación positiva entre el nivel educativo de los individuos y su propensión a emprender (Correa, Delgado \& Conde, 2011, p. 42).

Nadie puede dudar de la valentía que adiciona el gentilicio venezolano, sobre todo su juventud ante en un clima de desmotivación producto de las carencias de alimentos, el discurso político, malos servicios públicos, la separación de familiares producto de la migración, pero, posiblemente dicha actitud de coraje ante tales adversidades pudiera ser importante para muchos, pero no la necesaria, ya que si no se promueve un nuevo sujeto social a través de la construcción de un proyecto vida madurado desde su formación universitaria, la poca o mediana esperanza que pudiera existir se convertirá en desesperanza u otro clima más hostil.

El mundo natural se hace cada vez más insuficiente, la producción de alimentos o las condiciones necesarias para la vida confortable requieren cada vez de más esfuerzo, al mismo tiempo hay menos producción que tiene que ser repartida entre más personas. Es cierto que hay algunas personas que han acumulado riquezas, pero los seres humanos en su mayor parte vivimos en el borde de la escasez; tenemos que trabajar continuamente para subsistir; estamos permanentemente enfrentados a la amenaza de que las condiciones de vida que tenemos ahora pueden acabarse: o porque cambian las condiciones de trabajo y no hay adonde ir, o porque estamos envejeciendo. Perdemos la seguridad en el presente y vemos el futuro como una amenaza (Maturana, 1992, p. 62). 
La formación para el emprendimiento productivo ya no resulta una alternativa, sino una necesidad. Las universidades no pueden estar rezagadas ante estas exigencias de construir lo nuevo ante los desactualizados contextos que frenan la germinación de lo necesario.

La estructura académica está rezagada con respecto a las exigencias que plantean el emprendimiento y los procesos de innovación. Ambos aspectos, son asumidos sustratos de la realidad que rodea la formación profesional y los tópicos para afianzar la incubación de la innovación. De allí, que es importante establecer las bases en las que la estructura, involucraría a ambos referentes al interior del quehacer académico y los estímulos directos para la innovación (Gutiérrez, Asprilla \& Gutiérrez, 2014, p. 146).

La deserción estudiantil puede evaluarse de una manera multidisciplinaria, pasando desde la migración de los estudiantes al extranjero, la desmotivación por la situación del país, el antagonismo entre el planteamiento de la academia y las expectativas del educando. Tampoco es descartable son las razones financieras que obligan al estudiante a la búsqueda de actividades económicas para responder a sus gastos personales y familiares. Para Albarrán (2019), "un fenómeno muy complejo que ha puesto de manifiesto, en la población estudiantil, la falta de recursos económicos necesarios para la continuación de sus estudios, pues al verse limitados optan por trabajar o emigrar, ausentándose de las instituciones de enseñanza profesional” (p. 61). En esa línea,

el factor económico es uno de los principales motivos de deserción. La decisión de desertar en referencia a este factor, se asocia a la percepción del estudiante de ser capaz o no de solventar los costos universitarios. La situación que se presentó para estos jóvenes, fue la necesidad de escoger y terminar por abandonar los estudios para poder trabajar (Ferrer, Urribarrí \& Vera, 2017, p. 56).

Es necesario que las universidades puedan elaborar una propuesta de formación para el emprendimiento productivo. Sostiene Zamora (2017) que "la capacidad emprendedora de la sociedad, la educación, la apertura del mercado, las normas sociales y culturales, entre otros aspectos son los que posibilitan la generación de actividades productivas en el país, mientras que las regulaciones en el mercado de trabajo, la burocracia y la corrupción son elementos que entorpecen el dinamismo del sector productivo".

En tal sentido, mucho es lo que se puede lograr si la didáctica implementada en las universidades no solo se enfoque en el programa analítico o en el material bibliográfico, sino en los diversos factores que deben adicionar la acción educativa: la experiencia del educando, el entorno y la realidad social.

Cuando la dedicación en las aulas es únicamente docente, con una alta ausencia de análisis investigativo o proyección dirigida a la formación de investigadores o por lo menos intérpretes de dicha realidad, que aún no tiene cabida en las aulas por el ímpetu mecánico con que se plantea el desarrollo temático y reproductivo, la esperanza sigue estando puesta en la obtención de un título que contribuya a la ubicación laboral o disposición de empleo (Gutiérrez, Asprilla \& Gutiérrez, 2014, p. 148). 
Por ello, la acción formativa debe estar enmarcada en la construcción de la sociedad que se aspira, no se puede aislar la educación de los fines de bienestar económico, político y ciudadano. Por consiguiente, plantear un nuevo modelo educativo (enmarcado en los intereses de la sociedad) repercutiría en despertar el interés formativo de los educandos, así como la cultura de emprendimiento. Ambos factores facilitarían la llagada de los cambios que demanda la sociedad.

\footnotetext{
El emprendimiento promueve el progreso local en el ámbito social, económico, político, educativo entre otros, logrando influir en la dinámica productiva del entorno, la existencia de espíritu emprendedor, junto con la capacidad que tenga una sociedad para producir, asimilar cambios, que sean favorables para crecimiento económico (Del Valle, Meriño \& Martinez, 2018, p. 119).
}

No obstante, la búsqueda por promover una formación del emprendimiento desde los fundamentos de la andragogía permitirá tener una visión crítica de la realidad actual, en consonancia con la visión prospectiva del modelo de país que se quiere. Maturana (2001) refiere que "uno no puede reflexionar acerca de la educación sin hacerlo antes o simultáneamente acerca de esta cosa tan fundamental en el vivir cotidiano como es el proyecto de país en el cual están inmersas nuestras reflexiones sobre educación” (p. 4).

Si bien no escapan de la crisis (la desatención que sufren por parte del Estado pueden catalogarlas también como "víctimas"), las universidades no pueden mantener su pasividad académica y seguir ejerciendo el papel de espectadoras de esta situación. Como planteara Zemelman (2006), "no hay un espacio privilegiado para construir la historia, la historia se construye desde cualquier espacio todos los días, y si no, la construyen otros" (p. 40).

\section{Fuentes consultadas}

Abad, M. (2014). El emprendimiento, una herramienta para el combate contra la crisis y depresión económica. Recuperado de http://www.eumed.net/ce/2015/1/ emprendimiento.html

Albarrán, J. (2019). La deserción estudiantil en la Universidad de Los Andes (Venezuela). Educación y Humanismo, 21(36), 60-92. doi: http://dx10.17081/ eduhum.21.36.2806

Alcalá A. (2012) La praxis andragógica en los adultos de edad avanzada. Recuperado de http://postgrado.una.edu.ve/andragogia/paginas/ alcala2000.pdf

Ander Egg, E. (1977). Hacia una pedagogía autogestionaria. Buenos Aires: CID Editor.

Benavente, H. (2018) Oportunidades de emprendimiento en la Venezuela de hoy. Recuperado de https:/www.analitica.com/emprendimiento/oportunidades-deemprendimiento-en-la-venezuela-de-hoy/ 
Caraballo, R. (2012). La andragogía en la educación superior. Investigación y Postgrado, 22(2), 187-206. Recuperado de http://www.scielo.org.ve/scielo.php?script=sci arttext\&pid=S1316-00872007000200008\&lng=es\&tlng=es.

Correa, Z., Delgado, C.\& Conde, Y. (2011). Formación en emprendimiento en estudiantes de la carrera de administración de empresas en la Universidad Pública de Popayán. Revista Escuela de Administración de Negocios, 71, 40-51. Recuperado de https:// www.redalyc.org/pdf/206/20623157004.pdf

De Sousa, B. (2007). La universidad en el siglo XXI. Para una reforma democrática y emancipatoria de la universidad. La Paz: CIDES-UMSA, ASDI, Plural editores. Recuperado de http://www.boaventuradesousasantos.pt/media/universidad_ siglo_xxi-.pdf

Del Valle, Y., Meriño, V. \& Martinez, C. (2018). Emprendimiento sostenible: una opción para el crecimiento local. I+DRevista de Investigaciones, vol. 11(1), 116-128.

Delors, J. (1996) (Comp.). La educación encierra un tesoro. Informe a la UNESCO de la Comisión Internacional sobre la educación para el siglo XXI. Recuperado de http://www.unesco.org/education/pdf/DELORS_S.PDF

Panorama (2017). La carta que el artista plástico Carlos Cruz-Diez envió a los venezolanos. Recuperado de https://www.panorama.com.ve/espectaculos/La-carta-que-elartista-plastico-Carlos-Cruz-Diez-envio-a-los-venezolanos-20170428-0116.html

Doubront, M. (4 de diciembre de 2017). Educar para el emprendimiento no es una opción sino una necesidad. Recuperado de http://otrasvoceseneducacion.org/ archivos/259077

Fergusson, A. \& Lanz, R. (2011). La transformación universitaria y la relación universidad-Estado-mundo. Recuperado de http://w2.ucab.edu.ve/tl_files/HomeImages/noticias/alex \%20fergusson, $\% 20$ rigoberto\%20lanz.pdf

Ferrer, Y., Urribarrí, D. \& Vera, L. (2017). Factores que influyen en la deserción de los estudiantes de la Facultad de Ciencias Económicas y Sociales de La Universidad del Zulia (tesis de licenciatura). Universidad del Zulia, Maracaibo, Venezuela. Recuperado de https://www.aacademica.org/programa.cambio.socialcesaluz/19.pdf

Fowks, J. (19 de diciembre de 2018). Crece la tasa de desempleo juvenil en Latinoamérica. Recuperado de https://www.eltiempo.com/economia/sectores/informe-de-la-oitsobre-desempleo-juvenil-en-latinoamerica-307200

González Velasco, J. (2012). Teoría educativa transcompleja (Vol. I). Editorial Académica Española. Recuperado de http://200.7.170.212/portal/images/documentos/teoria educatica_transcompleja.pdf 
Gutiérrez, F. (1974). Pedagogía de la comunicación. San José Costa Rica: Editorial Costa Rica.

Gutiérrez, J., Asprilla, E. \& Gutiérrez, J. (2014). Emprendimiento e investigación en la escala de la formación profesional y la innovación empresarial en Colombia. Revista Escuela de Administración de Negocios, 76, 144-157. Recuperado de http://www.scielo.org.co/pdf/ean/n76/n76a09.pdf

Herrera, Z. (2019). La gestión del conocimiento en las Universidades experimentales de Venezuela. Revista Scientific, 4(11), 380-395. Recuperado de http://www.indteca. com/ojs/index.php/Revista_Scientific/article/view/314

Maturana, H. (1992). El sentido de lo humano. Santiago de Chile: Ediciones Pedagógicas Chilenas.

Maturana, H. (2001). Emociones y lenguaje en educación y política. Santiago de Chile: Dolmen. Recuperado de http://turismotactico.org/proyecto_pologaraia/wpcontent/uploads/2008/01/emociones.pdf

Morín, E. (1998). Introducción al pensamiento complejo. Barcelona: Gedisa.

Organización Internacional del Trabajo, OIT (2017). Empleo juvenil en América Latina y el Caribe. Recuperado de https:/www.ilo.org/americas/temas/empleo-juvenil/ lang--es/index.htm

Ortega y Gasset, J. (2001). Misión de la Universidad. Con indicaciones y notas para los cursos y conferencias de Raúl J. A. Palma. Recuperado de http://www.esi2. us.es/ fabio/mision.pdf

Petit, E. (2007). Enterprising Innovative Management as a Catalyst for Economic Enterprise. Revista de Ciencias Sociales, 13(3), 495-506. Recuperado de http://www.scielo. org.ve/scielo.php?script=sci_arttext\&pid=S1315-95182007000300010\&lng=es \&tlng=en.

Raffino, M (2019). Concepto de emprendimiento. Recuperado de https://concepto.de/ emprendimiento/

Rodríguez, A. (2014). Emprendimiento venezolano: mucho entusiasmo, pero falta formación. Recuperado de http://www.iesa.edu.ve/inicio/2014-marzo31/1774=emprendimiento-venezolano-mucho-entusiasmo,-pero-falta-formacion

Universidad Arturo Michelena (2019). Existe alta tasa de emprendimiento temprano en Venezuela. Recuperado de http://www.uam.edu.ve/actualidad/547-existe-altatasa-de-emprendimiento-temprano-en-venezuela.html 
Universidad Nacional Experimental Simón Rodríguez, UNESR (2009). Construcción colectiva de las políticas académicas y de investigación en el marco de las transformaciones en la UNESR para el fortalecimiento de la educación liberadora. Caracas: Vicerrectorado Académico UNESR.

Valdez, J. (2010). Andragogía: una lectura prospectiva. Caracas: Fundación Editorial El Perro y la Rana. Recuperado de http://josejimenez.com.ve/pnfae-jaa/archivos/ Andragogi\%CC\%81a\%20una\%20lectura\%20prospectiva\%20-\%20Julio\%20 Valdez.pdf

Vallmitjana, N. (2014). La actividad emprendedora de los graduados IQS (tesis doctoral). Universidad Ramón Llull, Barcelona, España.

Zamora-Boza, C. (2017). La importancia del emprendimiento en la economía: el caso de Ecuador. Revista Espacios, 39(7). Recuperado de https://www.revistaespacios. com/a18v39n07/a18v39n07p15.pdf

Zemelman, H. (2006). El conocimiento como desafío posible. México, DF: Instituto Politécnico Nacional. 